\title{
Modelling of the Radiological Contamination of the RBMK-1500 Reactor Water Purification and Cooling System
}

\author{
G. Poskas, ${ }^{1,2}$ R. Zujus, ${ }^{1}$ P. Poskas, ${ }^{1}$ and G. Miliauskas ${ }^{2}$ \\ ${ }^{1}$ Nuclear Engineering Laboratory, Lithuanian Energy Institute, 3 Breslaujos Street, 44403 Kaunas, Lithuania \\ ${ }^{2}$ Department of Thermal and Nuclear Energy, Kaunas University of Technology, 73 K. Donelaicio Street, 44029 Kaunas, Lithuania \\ Correspondence should be addressed to G. Poskas; g.poskas@gmail.com
}

Received 5 February 2014; Accepted 21 August 2014; Published 31 August 2014

Academic Editor: Ali Hainoun

Copyright (c) 2014 G. Poskas et al. This is an open access article distributed under the Creative Commons Attribution License, which permits unrestricted use, distribution, and reproduction in any medium, provided the original work is properly cited.

\begin{abstract}
This paper presents modelling results on the RBMK-1500 reactor water purification and cooling system (PCS) components contamination at Ignalina NPP Unit 1. The modelling was performed using a computer code LLWAA-DECOM (Tractebel Energy Engineering, Belgium), taking into consideration PCS components characteristics, parameters of the water flowing in circuits, system work regimes, and so forth. During the modelling, results on activity of PCS subsystems and components' deposits and nuclide composition of deposits at the moment of the final shutdown of the reactor, as well as activity decay of the most contaminated PCS components' deposits and dose rates after the final shutdown of the reactor, were obtained. Significant difference of contamination levels was revealed among PCS subsystems and subsystems components. The subsystem of nonpurified water is the most contaminated in PCS, and the activity of the least contaminated component in this subsystem is only $1.42 \%$ compared to the activity of the most contaminated component. The most contaminated and the least contaminated components of the purified water subsystem comprise $28.33 \%$ and $0.86 \%$ of activity, respectively, compared to the activity of the most contaminated PCS component.
\end{abstract}

\section{Introduction}

On December 31, 2009, after the final shutdown of Unit 2 (Unit 1 was closed down on December 31, 2004), Ignalina Nuclear Power Plant (INPP) terminated generation of electricity. After that the decommissioning is its main activity. In order to plan the dismantling process, data about the contamination levels, nuclide composition, amount of the decommissioning radioactive waste, and so forth, are necessary. Contamination of the systems components with radioactive particles is a result of the contaminated cooling liquid (water at INPP) circulating within these systems.

There are several possibilities for the main circulation circuit (MCC) and purification and cooling system (PCS) water to be contaminated with activation products [1]. But usually corrosion of the components is the most important problem. Intensity of corrosion in the reactor components depends on many factors, for example, temperature, composition of components and duration of operation, coolant flow regime, and so forth. Formation process of corrosion products and transfer mechanisms are described in detail in [2-5].

The corrosion products of systems components are transferred by the coolant to the reactor active zone. Here they form deposits on the surfaces of the equipment and are activated by high neutron fluxes. Due to erosion, these activated particles pass to the coolant, and due to its circulation they are deposited on the surfaces of the equipment located outside the reactor's active zone. Corrosion particles may not deposit on the walls of the equipment in the active zone, but they are activated during circulation through the active zone and form a layer of contaminated deposits on the surfaces of the equipment located outside the boundaries of the active zone [6]. Corrosion and erosion products of the activated equipment located in the active zone are another source for contamination of the equipment. In case there are defects in fuel rods, fission products in the nuclear fuel can migrate through damaged fuel claddings and pass to the coolant flowing in MCC. Fission products are also additionally 


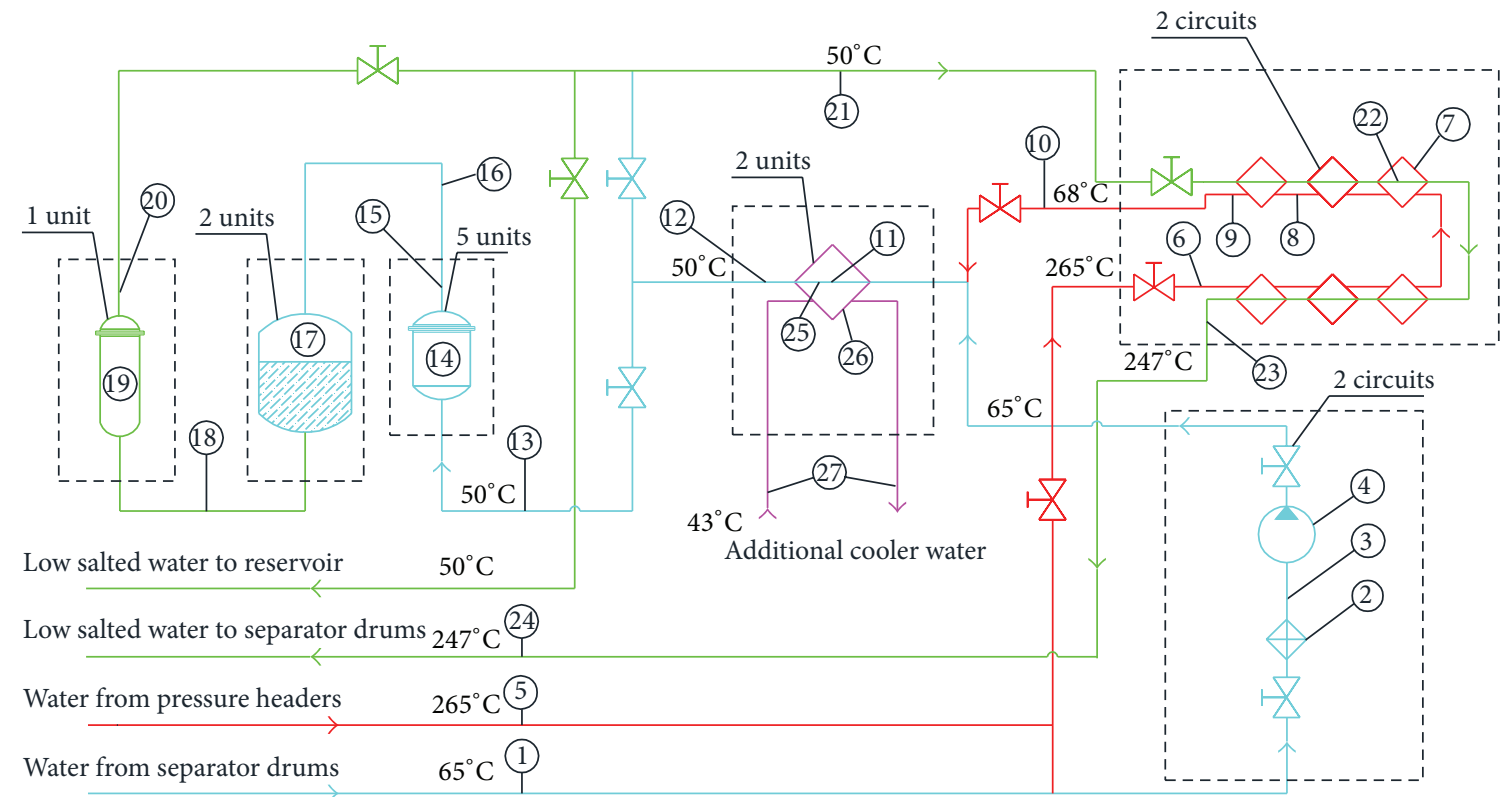

FIGURE 1: Simplified PCS scheme: 1-17: subsystem of nonpurified water, 18-24: subsystem of purified water, and 25-27: subsystem of additional cooling water.

generated during the fission of "tramp" uranium, whose particles are deposited on the outside of the fuel components' claddings (contaminated during manufacturing of the fuel). During operation, the coolant could be contaminated with these particles too [7]. Spectrum of the fission products of the "tramp" uranium is analogous to the spectrum of fission products in nuclear fuel. Radioactive contamination of the components is surface contamination since the activated particles deposit on the surfaces of the equipment.

The paper provides modelling and analysis results on activity of the PCS components at Ignalina NPP Unit 1 and deposits nuclide composition as well as variation of the most contaminated PCS components' deposits activity and their emitted dose rate after the final shutdown of the reactor.

\section{Purification and Cooling System (PCS)}

PCS maintains the quality of the water in the main circulation circuit as it cleans up the MCC water from corrosion products and soluble salts and controls radioactivity level in the MCC water. Moreover, PCS supplies water to the reactor in case of a shutdown or an emergency, washing MCC system during maintenance of the reactor, and saves precooled water to $50^{\circ} \mathrm{C}$ in a water-storage tank for start-up of the reactor [8]. Considering contamination of water, the PCS can be classified into three subsystems: nonpurified water, purified water, and additional cooling water. Figure 1 demonstrates a simplified technological scheme of PCS.

PCS consists of pipes from separator drums and pressure headers $(1,5)$, two mechanical filters in front of the pumps (2), two pumps (4), two regenerators each having six bodies (7), two additional cooler blocks (26), five cartridge (perlite) filters (14), two mix bed (ion-exchange) filters (17), and a filter trap (19).
During normal operation of the reactor, a part of MCC water is supplied to PCS (5) from MCC pressure headers and passes to the regenerators $(7,8)$ where it is cooled from $265^{\circ} \mathrm{C}$ to $68^{\circ} \mathrm{C}$ by the flow of the returning purified water. Operating temperature of the ion-exchange filters is up to $60^{\circ} \mathrm{C}$; thus the water of $68^{\circ} \mathrm{C}$ temperature is additionally cooled to $50^{\circ} \mathrm{C}$ in the additional cooling subsystem $(25,26,27)$. Further, water passes to the mechanical perlite filters (14) by the pipes (13, 15) where corrosion products are removed. Soluble salts and radioactive fission products are removed from the water in ion-exchange filters (17). The purified water is supplied again to the regenerators $(7,8)$ through the pipe $(21)$. Here it is preheated up to $247^{\circ} \mathrm{C}$ temperature by the water flowing from MCC pressure headers. Finally, it reaches the MCC separator drums through the pipe (24).

The main PCS components are also listed in Table 1.

\section{Methodology}

While modelling radioactive contamination of the reactor systems, there are a number of complicated processes (formation of contamination particles and their transfer and relaxation) that must be evaluated. During detailed analysis of the processes, complicated mathematical models and their numerical solution algorithms, requiring considerable calculation resources, are used. Due to this, simplifications are made while evaluating radioactive contamination of the systems in reactors; that is, empirical and semiempirical models and codes (PACTOLE [9], CRUDTRAN [10], ACEII [11], etc.) are used.

A computer code LLWAA-DECOM (Tractebel Energy Engineering, Belgium) was used for the modelling of radioactive contamination of PCS components. LLWAA-DECOM validation results are presented in [12]. It is demonstrated 
TABLE 1: Main PCS components.

\begin{tabular}{|c|c|c|}
\hline Number & Name of the component & Marking \\
\hline \multicolumn{3}{|c|}{ Subsystem of nonpurified water (NV-1) } \\
\hline 1 & Pipes from the MCC separator drum to PCS pumps & PCS-1 \\
\hline 2 & Mechanical filters in front of pumps (the body) & PCS-2 \\
\hline 3 & Parallel pipes to and from PCS pumps & PCS-3 \\
\hline 4 & PCS pumps CNR-500-115 (the body) & PCS-4 \\
\hline 5 & Pipes from MCC pressure headers to PCS pumps & PCS-5 \\
\hline 6 & Inlet pipes from pumps to regenerators & PCS-6 \\
\hline 7 & The regenerator (inner surface of the body) & PCS-7 \\
\hline 8 & The regenerator (outer surface of the pipes) & PCS-8 \\
\hline 9 & Pipes from regenerators to the additional cooler & PCS-9 \\
\hline 10 & The pipe from the regenerator to the additional cooler & PCS-10 \\
\hline 11 & Additional cooler (inner surface of pipes) & PCS-11 \\
\hline 12 & Pipes from the additional cooler to the water purification equipment & PCS-12 \\
\hline 13 & Pipes from the additional cooler to filters & PCS-13 \\
\hline 14 & The cartridge filter (the body) & PCS-14 \\
\hline 15 & Pipes to cartridge filters & PCS-15 \\
\hline 16 & The pipe to mix bed (ion-exchange) filter & PCS-16 \\
\hline 17 & The mix bed (ion-exchange) filters (the body) & PCS-17 \\
\hline \multicolumn{3}{|c|}{ Subsystem of purified water (NV-2) } \\
\hline 18 & The pipe between mix bed filters and filter trap & PCS-18 \\
\hline 19 & The filter trap (the body) & PCS-19 \\
\hline 20 & Pipes from filter trap to regenerator & PCS-20 \\
\hline 21 & The pipe to regenerator & PCS-21 \\
\hline 22 & The regenerator (inner surface of the pipes) & PCS-22 \\
\hline 23 & Pipes of returning water to separator drum & PCS-23 \\
\hline 24 & The pipe of returning water to separator drum & PCS-24 \\
\hline \multicolumn{3}{|c|}{ Subsystem of additional water cooling $(\mathrm{NV}-3)$} \\
\hline 25 & The additional cooler (outer surface of pipes) & PCS-25 \\
\hline 26 & The additional cooler (the body) & PCS-26 \\
\hline 27 & Pipes to and from the additional cooler & PCS-27 \\
\hline
\end{tabular}

that there is rather good correlation between predicted and measured dose rates and deposited activities results. When performing modelling, the following input data are necessary:

(i) operation characteristics of the system (number of operating cycles, duration of operating cycles),

(ii) circulating water parameters (water $\mathrm{pH}$, temperature, average rate, and volumetric activity),

(iii) characteristics of contamination particles (density, diameter, and solubility),

(iv) design parameters of the system components (design materials, geometric dimensions of the component, and roughness of the walls), and so forth.

Nuclide activity variation of the deposits on the wall of the system component is described using the following equation [12]:

$$
\frac{d W_{i}}{d t}=K_{d} * C v_{i} *\left(1-f r s p r_{i}\right)-W_{i} *\left(K_{r}+\lambda_{i}\right),
$$

where $W_{i}$ is surface activity of $i$ th nuclide on the wall, $\mathrm{Bq} / \mathrm{m}^{2}$; $K_{d}$ is particles deposition rate, $\mathrm{m} / \mathrm{s} ; C v_{i}$ is volumetric activity of $i$ th nuclide in the flux, $\mathrm{Bq} / \mathrm{m}^{3}$; $f r s p r_{i}$ is soluble part of $i$ th nuclide in the circulating agent; $K_{r}$ is particle relaxation coefficient, $\mathrm{s}^{-1} ; \lambda_{i}$ is decay constant of $i$ th nuclide, $\mathrm{s}^{-1} ; t$ is time. Deposition and relaxation coefficients $\left(K_{d}, K_{r}\right)$ depend on coolant characteristics (flow rate, temperature, Reynolds number, etc.), characteristics of the system equipment (geometry, roughness of the inner walls, and friction coefficient), and characteristics of radioactive particles (particles density in the coolant, diameter).

In LLWAA-DECOM nuclide activity in the deposits and dose rate at the outer surface can be evaluated for one component during one calculation cycle. Based on PCS Scheme 27 components (Table 1) different characteristics were chosen for evaluation.

\section{Results}

Analysis of modelling results on PCS components contamination shows that the pipes from MCC pressure headers 


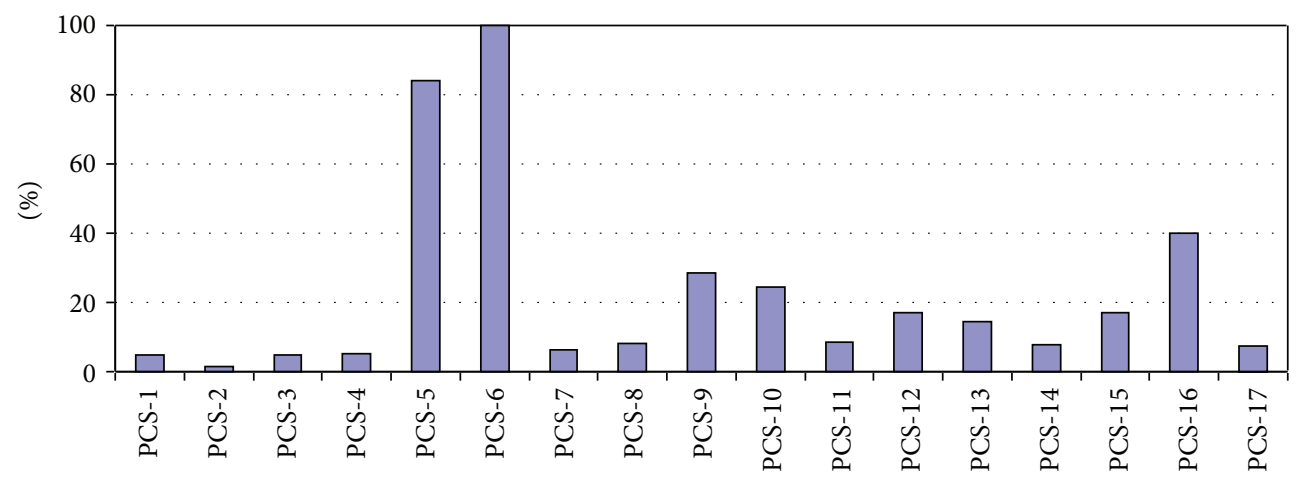

(a)

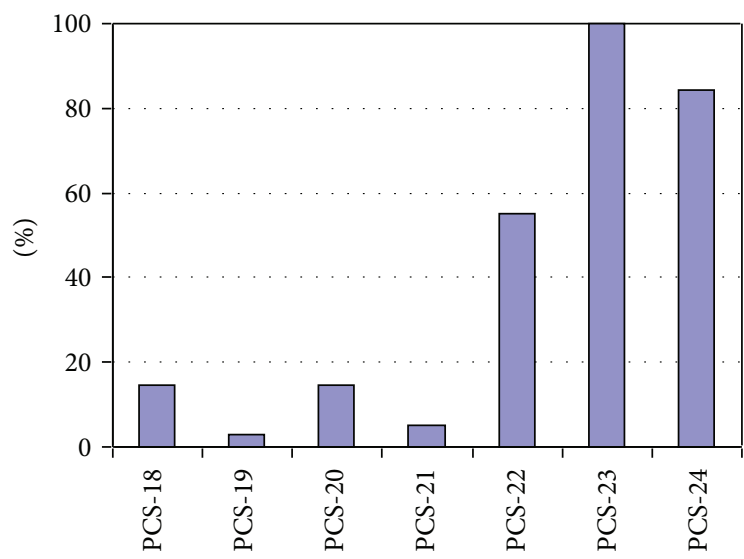

(b)

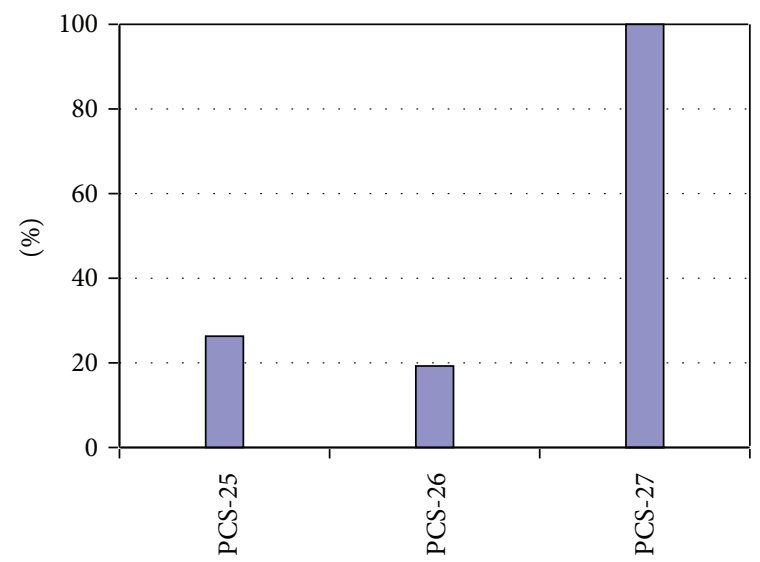

(c)

FIGURE 2: Relative activity of PCS components: (a) nonpurified water subsystem components (PCS-i/PCS-6), (b) purified water subsystem components (PCS-i/PCS-23), and (c) additional water cooling subsystem components (PCS-i/PCS-27).

to the PCS pumps (PCS-5) and also inlet pipes from the pumps to the regenerators (PCS-6) are the most contaminated components in the PCS nonpurified water subsystem (Figure 2(a)). The inner surface of the regenerator pipes (PCS-22) and the pipes of returning water to separator drum (PCS-23, PCS-24) are the most contaminated components in the PCS purified water subsystem (Figure 2(b)). In the additional water cooling subsystem of PCS, the pipes to and from the additional cooler (PCS-27) (Figure 2(c)) are the most contaminated components.

As Figure 2(a) demonstrates, there is a significant contamination level difference between PCS-5, PCS-6, and other PCS nonpurified water (NV-1) subsystem components. This is because the coolant temperature is higher in heat exchanger. Contamination level of PCS purified water (NV-2) subsystem components is lower (Figure 3(a)) because water passing through the filters is purified. Additional water cooling (NV3) subsystem contamination level is very low (Figure 3(a)) because the water of this subsystem does not mix directly with water of NV-1 and NV-2 subsystems. Some components (e.g., PCS-7 and PCS-22 or PCS-11 and PCS-26) in the heat exchanger have very different contamination levels on their inner and outer surfaces (Figure 2), since one surface is washed by contaminated water while the other contacts with purified or noncontaminated water.
Figure 3(a) shows that contamination of PCS subsystems and their components is very different. Activity of the deposits of the least contaminated component in the nonpurified water subsystem (NV-1) is only $1.42 \%$ compared to the activity of the most contaminated component. The most contaminated and the least contaminated components of the purified water subsystem (NV-2) comprise $28.33 \%$ and $0.86 \%$ of activity, respectively, compared to the activity of the most contaminated NV-1 component. Meanwhile, activity of the additional water cooling subsystem (NV-3) component comprises only 0.0005 per cent compared to activity of the most contaminated component of NV-1.

Figure 3(b) shows distribution of relative activity of MCC and PCS subsystems most and least contaminated components, compared to MCC characteristic component (main circulation pump activity). Analysis of the data [13] revealed that the contamination levels of MCC specific components in the reactor active zone and in the separator drums parts filled with water are the highest. So, for comparison purposes, the MCC main circulation pump was selected as it is a more typical component of this system. As can be seen, contamination levels are very different among MCC. The activity of the least contaminated component of MCC is only $7.44 \%$ compared to the contamination of the pump. The subsystem of nonpurified water (PCS NV-1) is characterised by $26.37 \%$ 


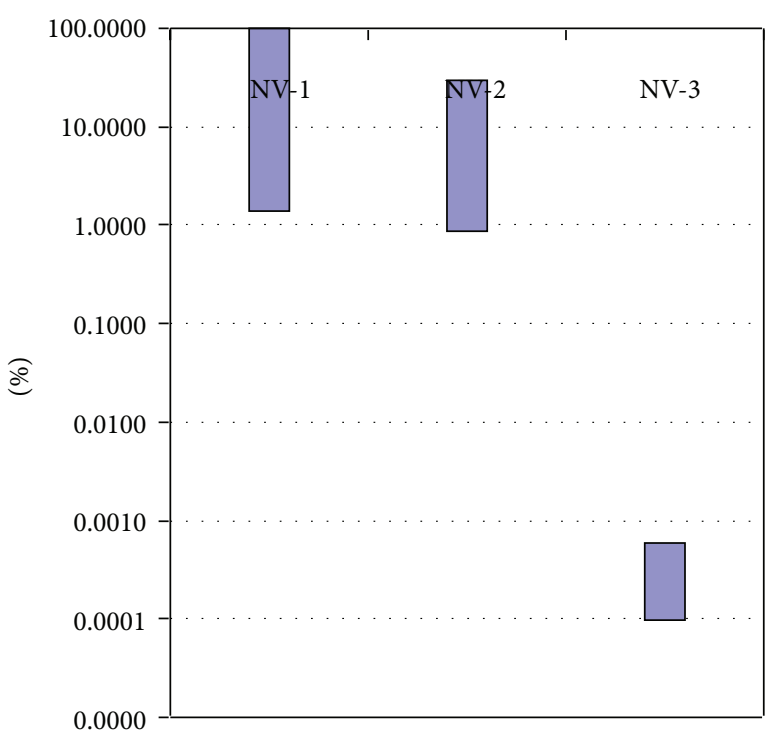

(a)

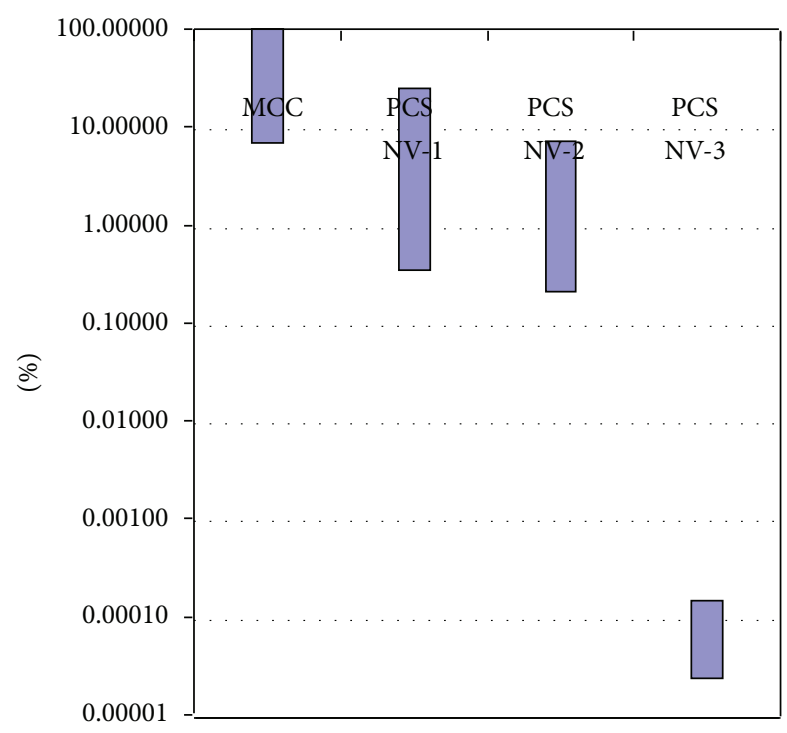

(b)

FIGURE 3: (a) Distribution of relative deposits activity of PCS subsystem components (PCS-i/PCS-6), (b) distribution of relative deposits activity on PCS subsystem components compared to the MCC main circulation pump activity (PCI-I/MCC pump).

relative activity for the most contaminated component and $0.37 \%$ relative activity for the least contaminated component compared to the contamination of the MCC main circulation pump. The most contaminated and the least contaminated components of the purified water subsystem (PCS NV-2) constitute $7.47 \%$ and $0.23 \%$ of activity, respectively, compared to the activity of the MCC main circulation pump. Meanwhile, activity of the additional water cooling subsystem (PCS NV-3) component constitutes only 0.00013 per cent compared to activity of the MCC main circulation pump.

As can be seen from Figures 4(a), 4(b), and 4(c), contamination of water is mostly determined by Cs-134 (PCS NV-1 and PCS NV-3 $31 \%$ and PCS NV-2 50\% of total contamination) and Cs-137 (PCS NV-1 and PCS NV-3 $\sim 22 \%$ and PCS NV-2 $\sim 35 \%$ of total contamination). The dominant nuclide in deposits is Fe-55: PCS NV-1 subsystem $\sim 56 \%-\sim 59 \%$ of total contamination (Figure 4(a)), PCS NV-2 subsystem $\sim 55 \%-\sim 56 \%$ of total contamination (Figure $4(\mathrm{~b})$ ), and PCS NV-3 subsystem $\sim 58 \%$ of total contamination (Figure $4(\mathrm{c})$ ). In the water, Fe-55 constitutes only $\sim 5 \%-$ $\sim 16 \%$ of total contamination. So there is a big difference in nuclide composition in water and in deposits (Figure 4). Such difference in nuclide distribution in water and deposits is determined by nuclide solubility in water. Only undissolved part of the nuclide deposits on the surface. Hence, Fe-55 concentration in deposits is considerably higher compared to Cs-134 and Cs-137, although Cs-134 and Cs-137 have higher concentrations in water than Fe-55. This is due to higher solubility of Cs-134 and Cs-137 in water compared to Fe-55. Cs-134 and Cs-137 species are deposited not only on PCS-23 (Figure 4) but on all other components as well. Their input to total activity for most cases is less than $1 \%$; therefore they are under the legend "Other." Moreover, PCS components PCS18 to PCS-24 are after filters (subsystem of purified water) and corrosion nuclides are filtered better than fission nuclides; therefore relative activity of Cs nuclides is increased.

The nuclide composition of deposits changes with time due to different nuclides decay. Figures 5(a) and 5(b) demonstrate nuclide composition variation of deposits on the walls of the two most contaminated components of the PCS subsystem. After 35 years, contamination of the PCS components is highly determined by a long-lived nuclide $\mathrm{Ni}-63$, since short-lived nuclides decay in a short time (Fe-55, Fe-59, Co58, Co-60, and Mn-54). The contribution of other long-lived nuclides to the total contamination increases also as their activity practically remains unchanged.

During dismantling, the most important nuclides are those that determine the dose rate by $\gamma$ radiation from contaminated components. The activity of short lived nuclides is significantly decreasing with time, so the dose rate is decreasing too (Figures 6 and 7). At the moment of the final shutdown of the reactor, $\gamma$ radiation from contaminated PCS components is mostly determined by Co-60, by Fe-59 and Mn-54, and by Co- 60 after 5 years after the shutdown (Figures 6(a) and 6(b)). After several decades contribution of Nb-94 and Cs-137 to the dose rate increases and contribution of Co60 decreases significantly. Although after some time Ni-63 has the biggest contribution to the activity of the deposits, it is a weak $\beta$-emitter; thus, Ni-63 has no practical influence on the dose rate.

Analysis shows that the contribution to the dose rate from the contaminated water in PCS is much lower compared to the contribution of the deposits. Thus, modelled and measured dose rate values can be compared without the need to remove the coolant from the system. The water is removed from the system prior to dismantling works.

As it is shown in Figure 7, the dose rate decreases significantly (by $\sim 70 \%$ ) after five years after the final shutdown of 


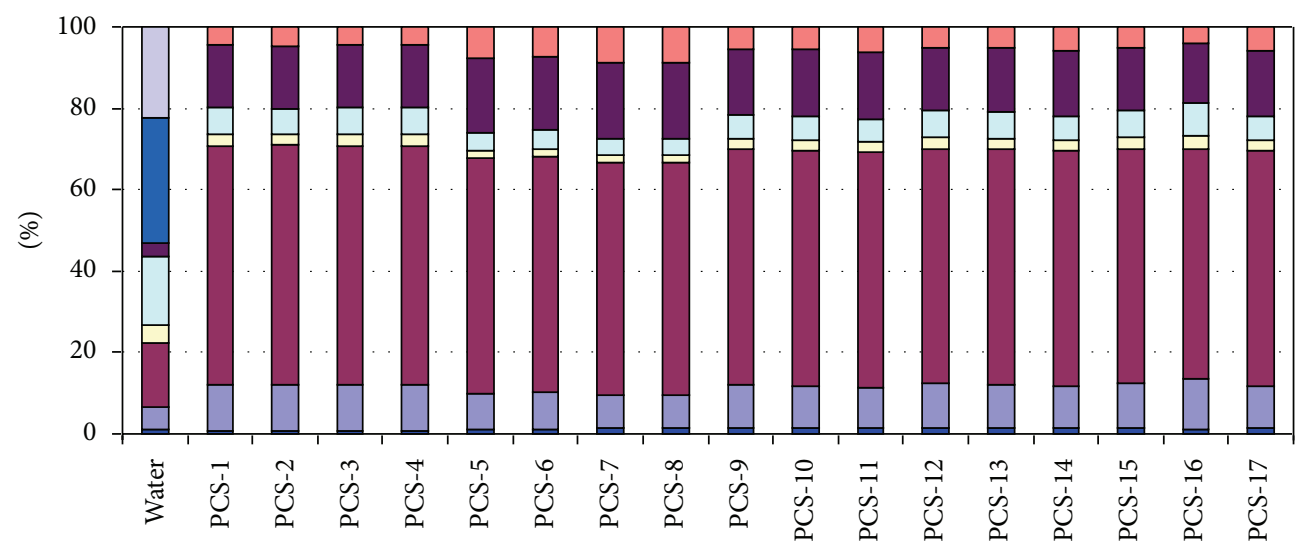

(a)

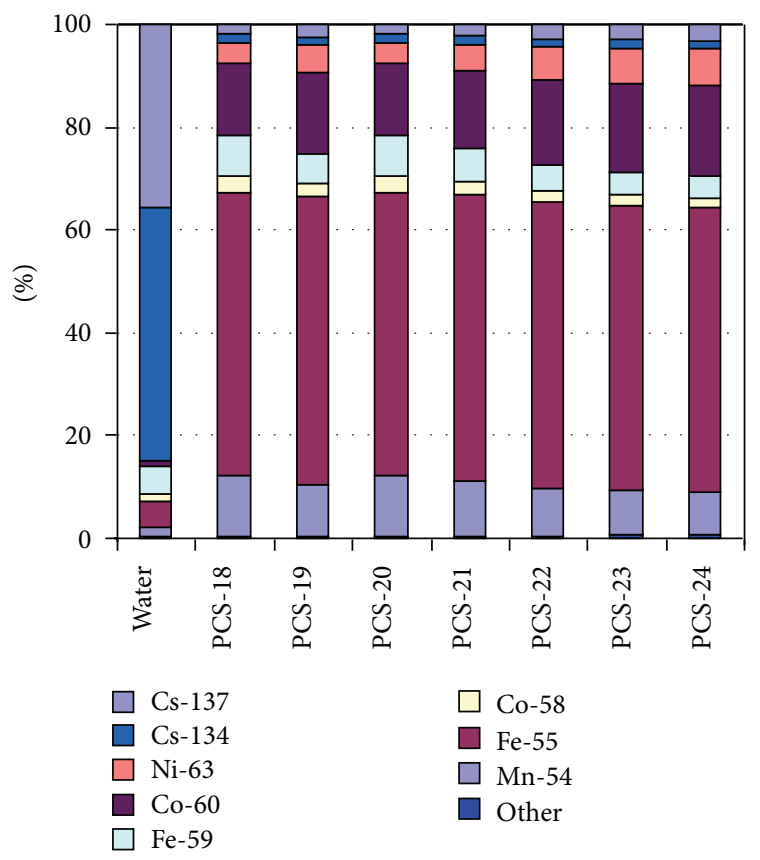

(b)

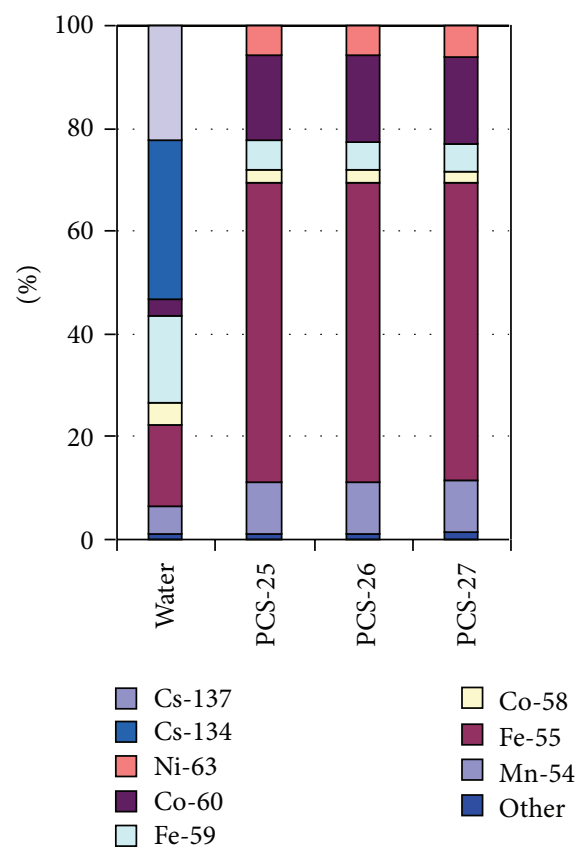

(c)

FIGURE 4: Nuclide composition of the deposits in the PCS: (a) nonpurified water subsystem, (b) purified water subsystem, and (c) additional water cooling subsystem (other $<1 \%$ ).

the reactor. This is caused by decay of short-lived radionuclides Fe-55, Co-58, and Mn-54. The PCS dismantling works will not start right after the final shutdown of Unit 1. This is related to the strategy of the spent nuclear fuel management. It will be transferred to the dry storage facility only, at least, after 5 years in the pools. Thus such significant decrease of dose rate will allow performing dismantling of the installations and radwaste management with lower radiation doses to the workers.

\section{Conclusions}

After analysis of the modelling results on the contamination of the water purification and cooling system (PCS) components, the following conclusions have been drawn.
(1) Contamination of PCS subsystems and their components is very different. Activity of the deposits of the least contaminated component in the nonpurified water subsystem (NV-1) is only $1.42 \%$ compared to the activity of the most contaminated component. The most contaminated and the least contaminated components of the purified water subsystem (NV-2) constitute $28.33 \%$ and $0.86 \%$ of activity, respectively, compared to the activity of the most contaminated NV-1 component. Meanwhile, activity of the additional water cooling subsystem (NV-3) component constitutes only 0.0005 per cent compared to activity of the most contaminated component of NV-1.

(2) Nuclide composition of deposits formed on the PCS components differs significantly from circulating 


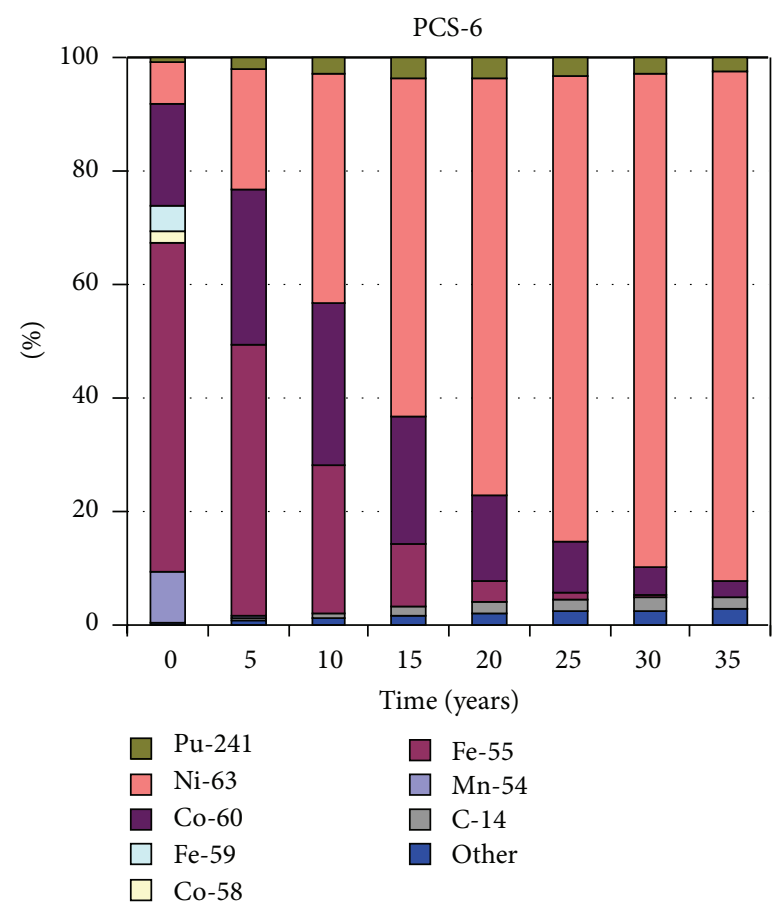

(a)

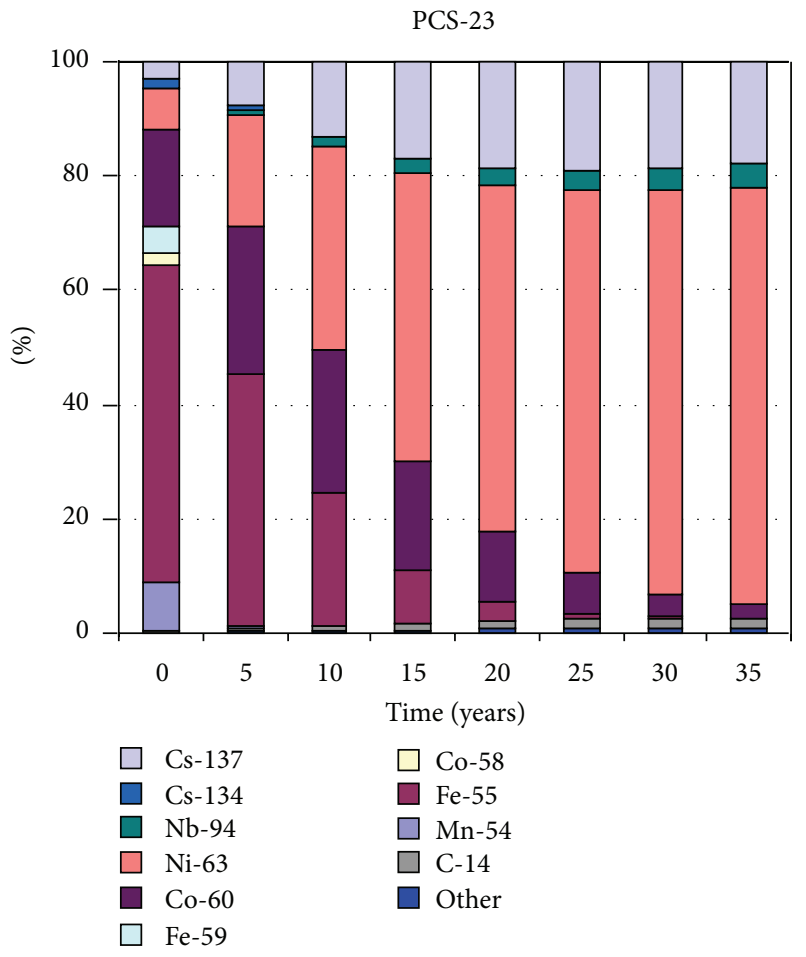

(b)

FIgURE 5: Activity variation of deposits of components with time: (a) PCS-6 and (b) PCS-23 (other $<1 \%$ ).

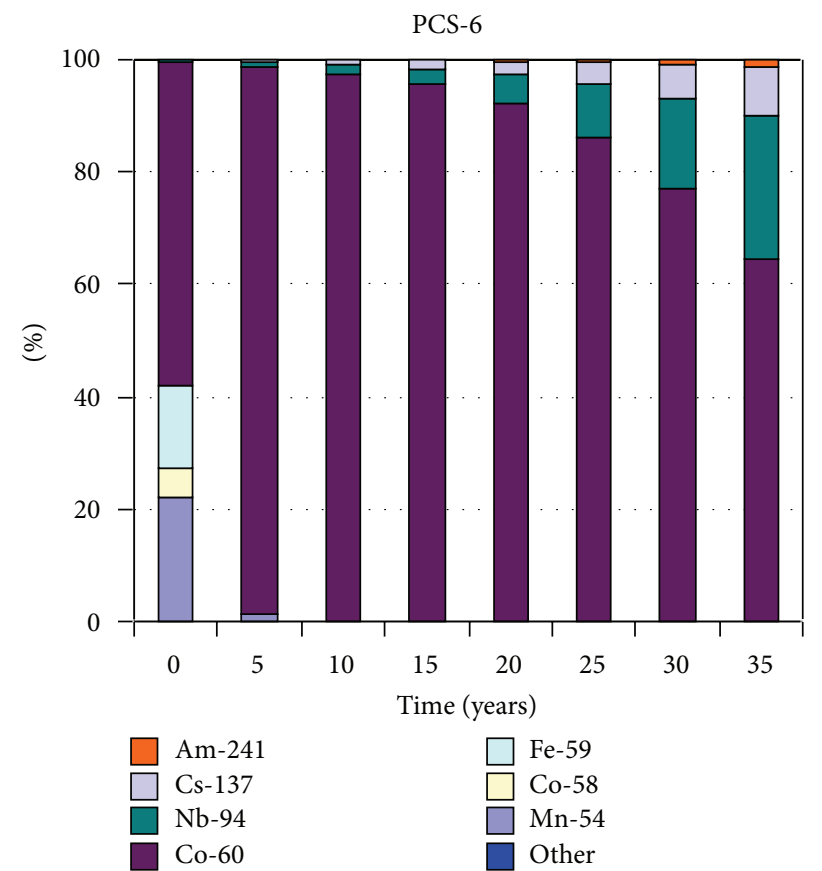

(a)

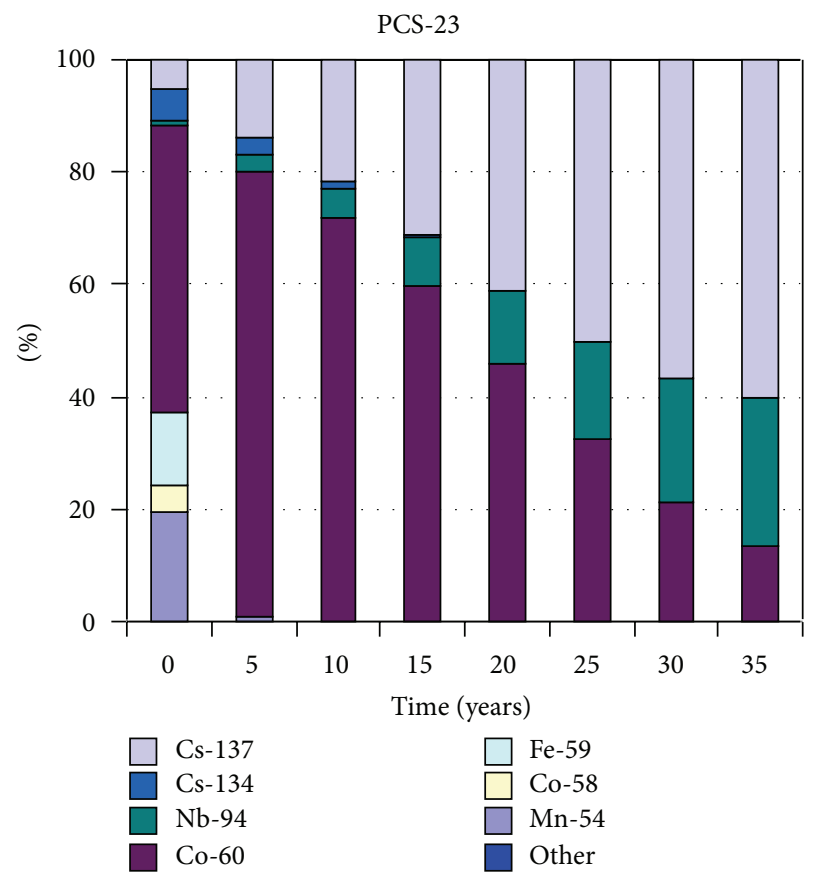

(b)

FIGURE 6: Variation of nuclide composition with time (other < 1\%): (a) PCS-6 component and (b) PCS-23 component. 


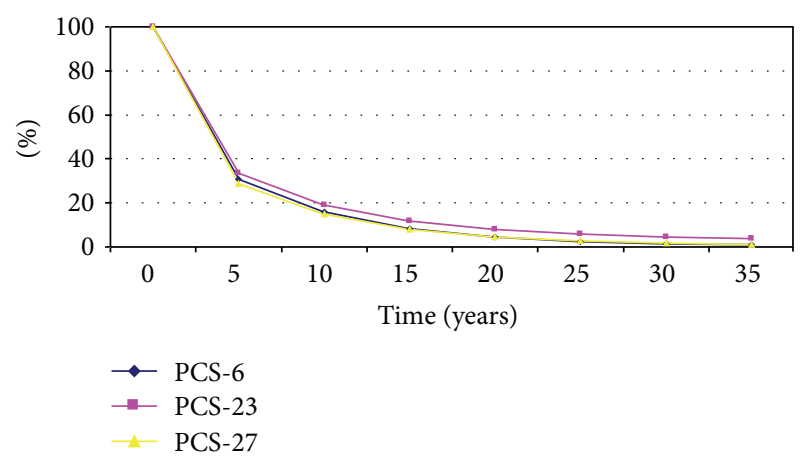

FIGURE 7: Variation of dose rate of some PCS components over time.

water nuclide composition. Cs-134 and Cs-137 are dominant nuclides in the water, while Fe-55, Co-60, and Mn-54 are dominant nuclides in the deposits.

(3) Short-lived radionuclides Fe-59 and Mn-54 have the biggest influence on the dose rate at the shutdown of the reactor; five years after the shutdown, Co-60 has the biggest influence. After several decades, $\mathrm{Nb}$ 94 and Cs-137 contribute mostly to the dose rate. Due to short-lived radionuclides decay the dose rate decreases significantly over time.

\section{Conflict of Interests}

The authors declare that there is no conflict of interests regarding the publication of this paper.

\section{References}

[1] IAEA, "Radiological characterization of shut down nuclear reactors for decommissioning purposes," Tech. Rep. 389, IAEA, Vienna, Austria, 1998.

[2] "Modelling of transport of radioactive substances in the primary circuit of water-cooled reactors," Technical Reports Series 1672, IAEA, Vienna, Austria, 2012.

[3] D. You, J. Lambert, and D. Feron, "Dissolution and solubility of cobalt and nickel ferrites in PWR primary conditions," in Proceedings of the 7th International Conference on Water Chemistry of Nuclear Reactors Systems, Bournemouth, UK, 1996.

[4] V. G. Kritsky, Water Chemistry and Corrosion of Nuclear Power Plant Structural Materials, Sinto, 1996.

[5] "Coolant technology of water cooled reactors: an overview," Tech. Rep. 347, IAEA, Vienna, Austria, 1993.

[6] M. Laraia, Ed., Nuclear Decommissioning: Planning, Execution and International Experience, vol. 36 of Woodhead Publishing Series in Energy, 2012.

[7] B. J. Lewis and A. Husain, "Modelling the activity of 129I in the primary coolant of a CANDU reactor," Journal of Nuclear Materials, vol. 312, no. 1, pp. 81-96, 2003.

[8] K. Almenas, A. Kaliatka, and E. Ušpuras, Ignalina RBMK-1500: A Source Book, Ignalina Safety Analysis Group, 1998.

[9] P. Beslu, "A computer code PACTOLE to predict activation corrosion products in PWRs," in Proceedings of the International
Conference on Water Chemistry of Nuclear Reactor Systems, British Nuclear Energy Society, Bournemouth, UK, 1978.

[10] C. B. LEE, Modeling of corrosion product transport in PWR primary system [Ph.D. thesis], Massachusetts Institute of Technology, Cambridge, Mass, USA, 1990.

[11] A. K. Burrill and P. Menut, "A description of the activity transport computer codes in the IAEA benchmarking exercise," in Water Chemistry of Nuclear Reactor Systems 8: Proceedings of the Conference Organized by the British Nuclear Energy Society and Held in Bournemouth, UK, on 22-26 October 2000, vol. 1, 2000.

[12] B. B. Lemens, P. Centner, and B. K. Mannaerts, "Determination and declaration of critical nuclide inventories in Belgian NPP radwaste streams," in Proceedings of the Waste Management Conference (WM '99), pp. 1-14, March 1999, Manule de conception du programme de calcul de lactive dans depots des equipments des centrals de Doel et Tihange. CNTKCD/4NT/8317/000/01 Ed.990121.

[13] P. Poskas, R. Zujus, and A. Brazauskaite, "Preliminary radiological characterisation of the main circulation circuit at Ignalina NPP for decommissioning purposes," in Proceedings of the Waste Management Conference (WM '04), pp. 1-9, Tucson, Ariz, USA, March 2004. 


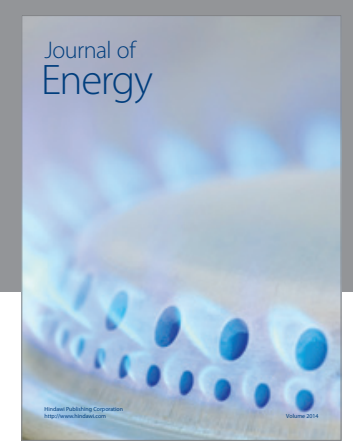

Journal of

Industrial Engineering
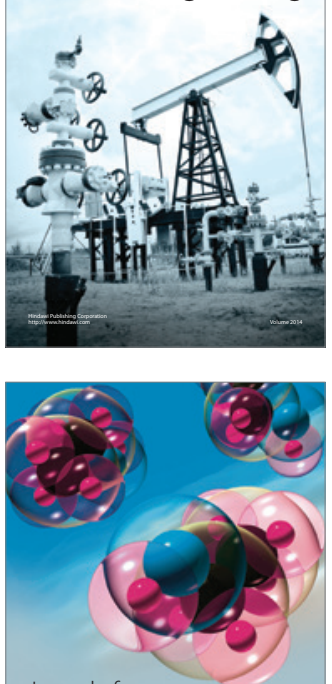

Fuels
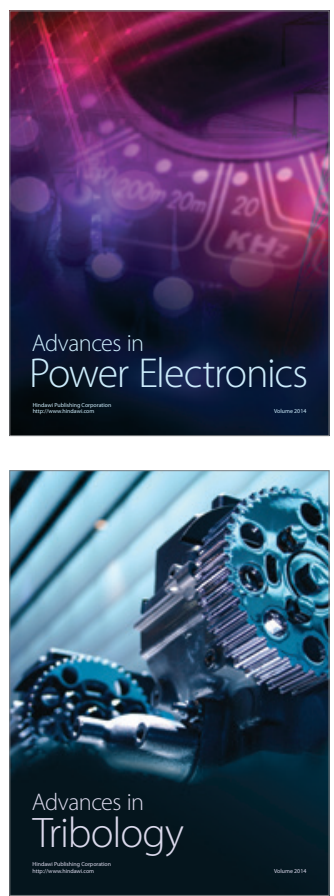

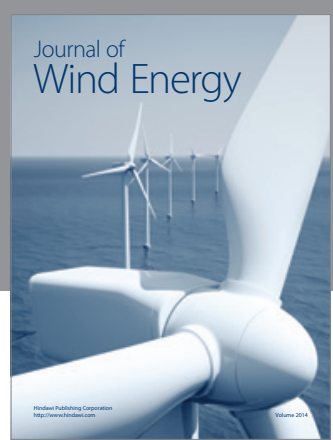

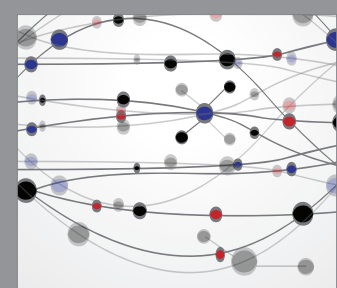

The Scientific World Journal

Submit your manuscripts at http://www.hindawi.com

Journal of

Structures
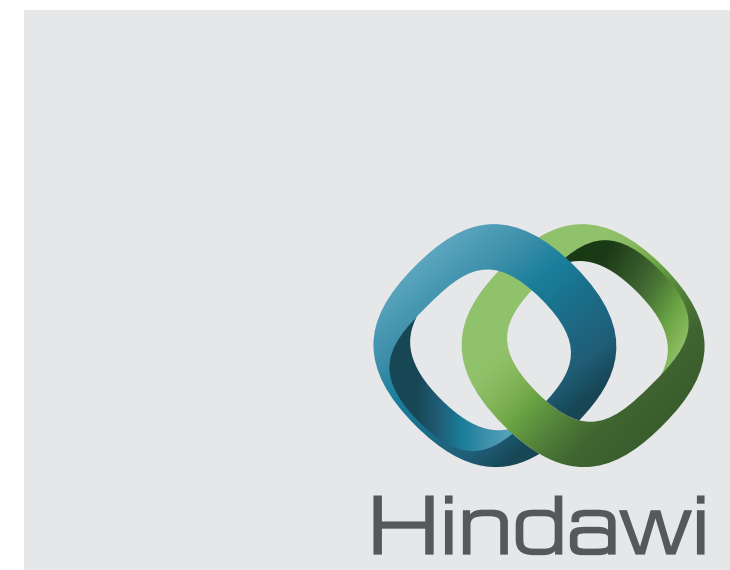

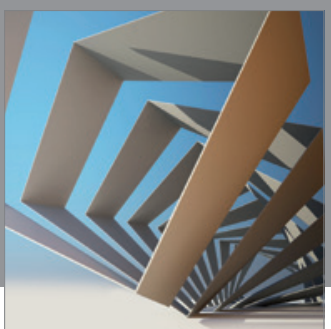

Rotating

Machinery
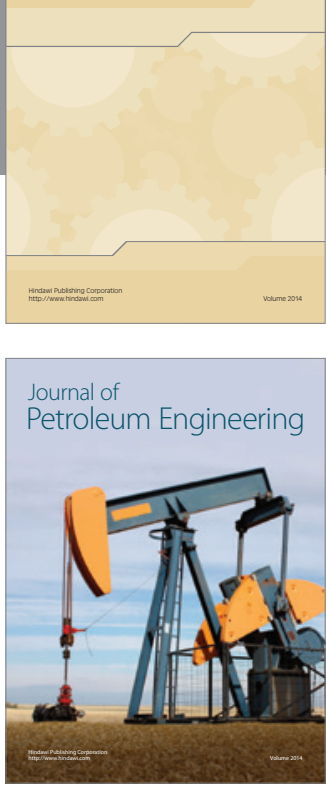

Journal of

Solar Energy
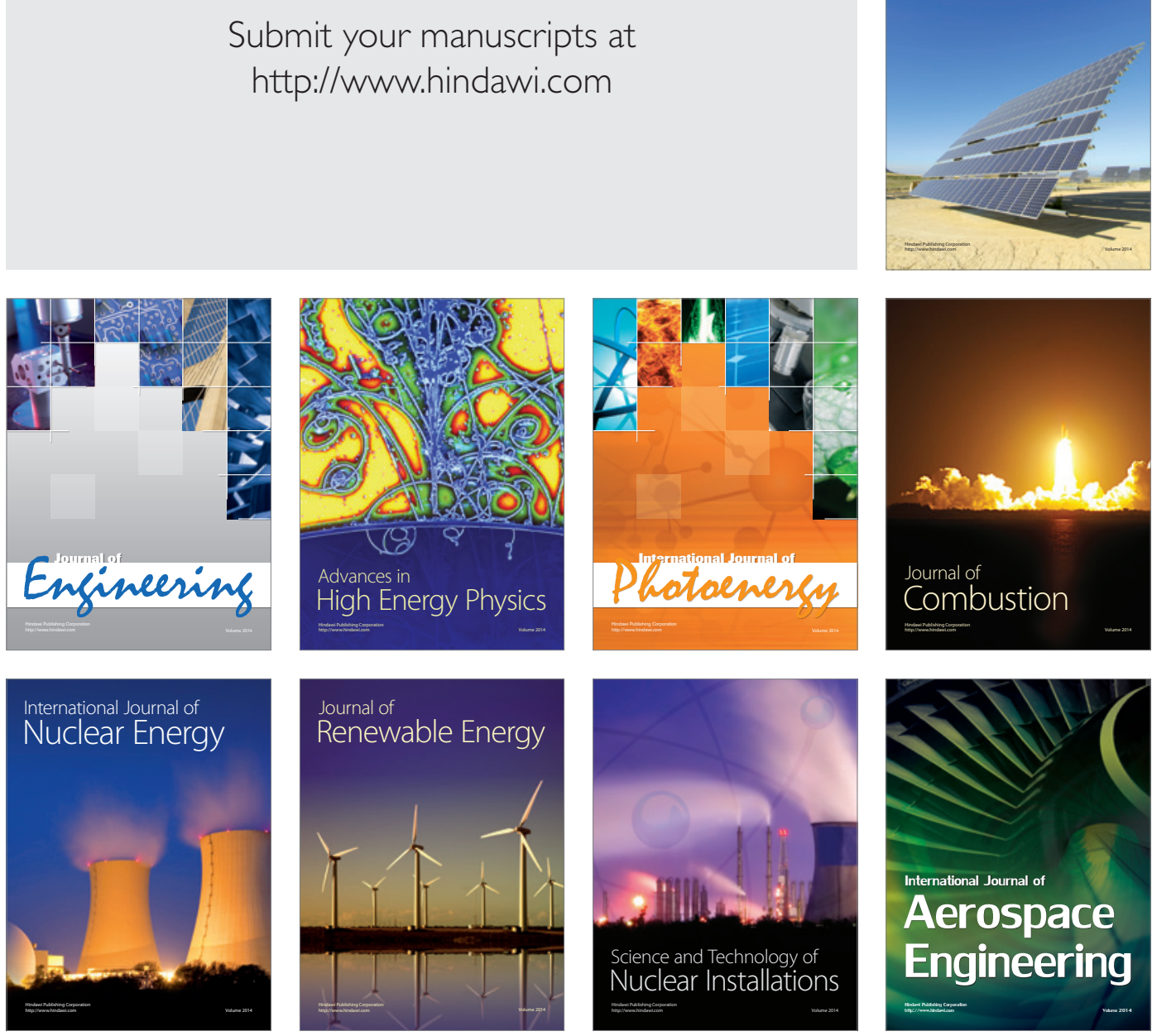